\title{
$\beta$-decay studies of $r$-process nuclei using the Advanced Implantation Detector Array (AIDA)
}

C.J. Griffin ${ }^{* a}$, T. Davinson ${ }^{a}$, A. Estrade ${ }^{a}$, D. Braga ${ }^{b}$, I. Burrows ${ }^{c}$, P. Coleman-Smith ${ }^{c}$, T. Grahn ${ }^{d}$, A. Grant ${ }^{c}$, L.J. Harkness-Brennan ${ }^{d}$, M. Kogimtzis $^{c}$, I. Lazarus ${ }^{c}$, S. Letts ${ }^{c}$,

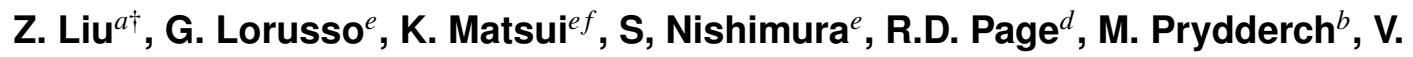
Pucknell $^{c}$, S. Rinta-Antila ${ }^{d}$, O. Roberts ${ }^{g}$, D.A. Seddon ${ }^{d}$, J. Simpson ${ }^{c}$, J. Strachan $^{c}$, S.L. Thomas ${ }^{b}$, P.J. Woods ${ }^{a}$

${ }^{a}$ School of Physics and Astronomy, University of Edinburgh, United Kingdom

${ }^{b}$ STFC Rutherford Appleton Laboratory, Detectors and Electronics Division, United Kingdom

${ }^{c}$ STFC Daresbury Laboratory, Nuclear Physics Group, United Kingdom

${ }^{d}$ Department of Physics, University of Liverpool, United Kingdom

${ }^{e}$ RIKEN Nishina Centre, RIKEN, Saitama, Japan

${ }^{f}$ Department of Physics, University of Tokyo, Tokyo, Japan

${ }^{g}$ School of Computing, Engineering and Mathematics, University of Brighton, United Kingdom

$\dagger$ Current address: Heavy Ion Research Facility in Lanzhou, Lanzhou, China

E-mail: c.j.griffin@ed.ac.uk

Creating around half of all isotopes heavier than iron, the $r$-process is an important process in nucleosynthesis and the chemical evolution of the Universe. However, the lack of experimental nuclear data for unstable isotopes is one important obstacle for a complete understanding of the $r$-process. Key parameters in $r$-process calculations include the $\beta$-decay half-lives and $\beta$ delayed neutron emission probabilities $P_{n}$ for neutron-rich isotopes along the $r$-process path. The Advanced Implantation Detector Array (AIDA) represents the latest generation of silicon implantation detectors for use at fragmentation beam facilities and will improve the precision and sensitivity of experiments, allowing the study of new and rare nuclear decay processes.

XIII Nuclei in the Cosmos,

7-11 July, 2014

Debrecen, Hungary

\footnotetext{
* Speaker.
} 


\section{Astrophysical Motivation}

The framework around which we base our present knowledge of nucleosynthesis is built upon the seminal works of Burbidge et al.[1] and Cameron[2], published in 1957. More recent work[3] has updated the foundations laid out in the 1950s, but it remains clear that the slow $[4,5]$ and rapid[6, 7] neutron-capture processes are key mechanisms in producing elements heavier than iron.

The rapid neutron-capture process ( $r$-process) is a nucleosynthesis mechanism in which a seed nucleus captures neutrons on a time scale much shorter than that for $\beta$-decay. It is thought to be responsible for the nucleosynthesis of around half of all isotopes heavier than iron and, as such, understanding the $r$-process is key to unlocking a deeper knowledge of the chemical evolution of the Universe. Requiring neutron densities $n_{n}>10^{20} \mathrm{~cm}^{-3}$ and temperatures of $T \sim 10^{9} \mathrm{~K}[6]$, the $r$-process is thought to take place within neutron star mergers and/or core-collapse supernovae. Complex models of these sites aim to predict the observed isotopic abundances of $r$-process nuclei, but fail to do so reliably due to large uncertainties in the input parameters.

Two key nuclear physics inputs used to constrain $r$-process nucleosynthesis models are the $\beta$-decay half-lives[8] and the probability of $\beta$-delayed neutron emission $P_{n}[9]$ of exotic nuclei.

$\beta$-decay half-lives determine the flow of matter to heavy isotopes and influence the shape of the abundance peaks. For example, longer-lived isotopes near neutron shell closures, exhibiting relatively long half-lives, become waiting point nuclei that block progression to more neutron rich isotopes, causing an 'accumulation' of matter in the longer-lived isotopes. The observed abundance peaks are then formed after their decay back to stability. $\beta$-delayed neutron $(\beta \mathrm{n})$ emission can occur in the decays of very neutron-rich nuclei, with a finite probability $P_{n}$, when the $\beta$-decay energy $Q_{\beta}$ exceeds the neutron separation energy $S_{n}$ in the daughter nucleus. $P_{n}$ values are thought to be another key parameter in determining final $r$-process abundances, thus, an accurate knowledge of both $\beta$-decay half-lives and $P_{n}$ values is key in understanding the nature of the $r$-process.

\section{The Advanced Implantation Detector Array}

The Advanced Implantation Detector Array (AIDA)[10] represents the latest generation of silicon implantation detectors for use in decay spectroscopy measurements of exotic nuclei at fragmentation beam facilities. Designed to improve upon current generation detectors used to measure heavy ion implantation-decay events, AIDA faces several challenges, requiring high detector pixelation and fast overload recovery, allowing accurate implantation-decay correlations over microsecond timescales. This section will take a closer look at key elements of the AIDA instrumentation.

Double-sided silicon strip detector (DSSSD) AIDA is designed for use at facilities capable of producing highly energetic particle beams with a wide range of isotopes and energies, each capable of passing though different volumes of silicon. To maximise the number of successful implants, AIDA is used with a stack of MSL type BB18 DSSSDs, which can be used in one of two configurations: an $8 \mathrm{~cm} \times 8 \mathrm{~cm}$ single wafer; or a $24 \mathrm{~cm} \times 8 \mathrm{~cm}$ triple wafer, created by bonding three single wafers along the rear $n-n$ ohmic strips. Each $8 \mathrm{~cm} \times 8 \mathrm{~cm}$ wafer has a thickness of $1000 \mu \mathrm{m}$ and is highly segmented, featuring 128 strips with a $625 \mu \mathrm{m}$ pitch in both the $x$ and $y$ directions. Multiple guard rings on both the $p-n$ and $n-n$ sides of the wafer reduce total leakage current to a typical value of $\sim 3.5 \mu \mathrm{A}$ at the operating bias of $\sim 200 \mathrm{~V}$. 


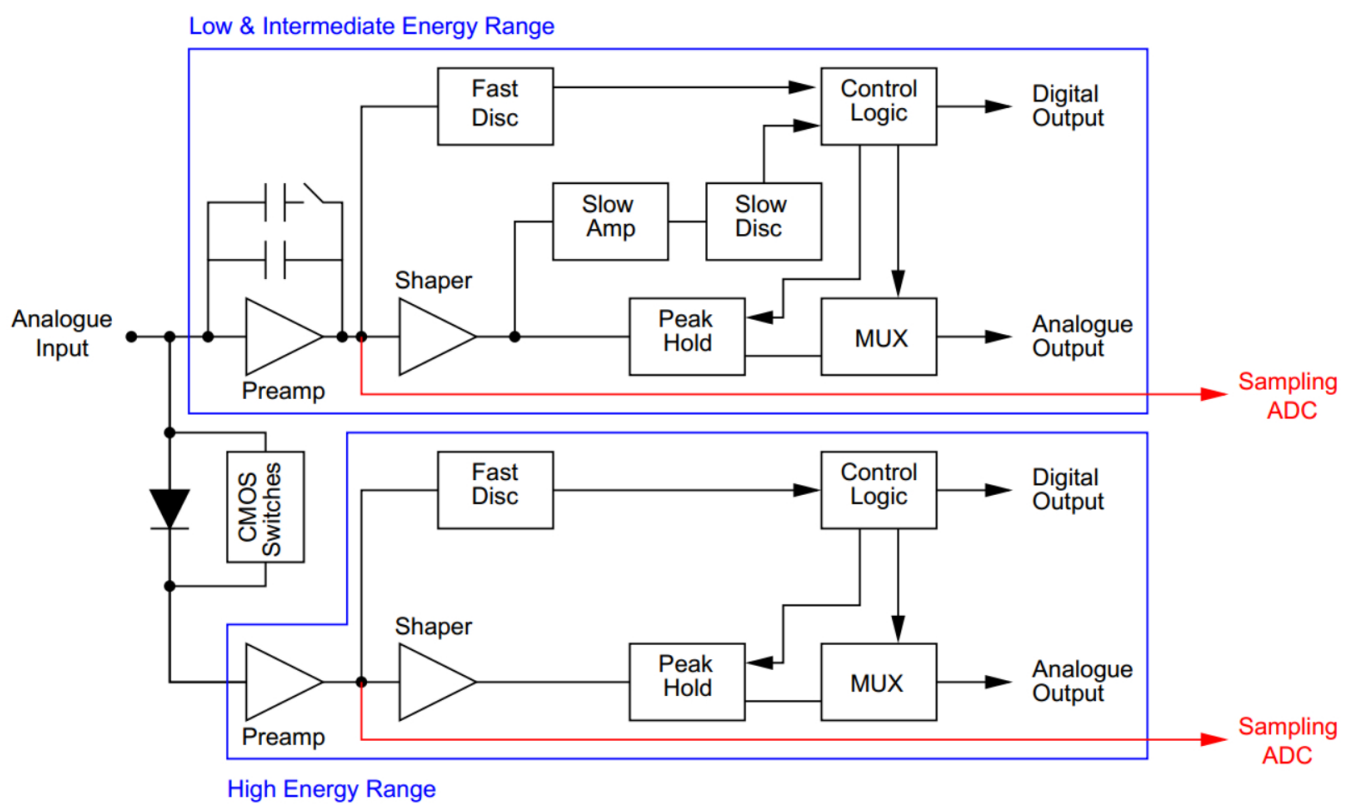

Figure 1: A schematic diagram showing the functionality of one ASIC channel. Visible are the two signal pathways available, depending on whether the energy deposited in the detector falls into the high or low energy range.

Signals are carried from the detectors to the Front End Electronics (see later paragraph) via flexible Kapton PCBs.

Implantation-decay correlation One of the challenges faced by AIDA is the accurate correlation of implantation-decay events. Exotic and highly energetic ( $100-200 \mathrm{MeV} /$ nucleon, A $\leq 240$ ) heavy ions are implanted in the DSSSD stack and subsequently undergo radioactive decay, emitting low-energy $\beta$ particles, protons, $\alpha$ particles, neutrons and $\gamma$ rays. The neutrons and $\gamma$-rays leave the silicon undetected, but the DSSSDs must be capable of detecting the other decay products with sufficient precision and accuracy to allow for correlations with the implantation event. High segmentation of the DSSSDs allows for higher implantation rates due to the reduced likelihood of multiple implants in one pixel within the lifetime of one species.

Application specific integrated circuit (ASIC) To enable the rapid measurement of implantation and decay events in a large number of strips, application specific integrated circuits (ASICs) with a high channel density are the only realistic solution for compact signal processing. The ASICs[11] are specifically designed to meet the needs of the task on timescales short enough to enable the decay half-lives to be accurately measured. One ASIC can process 16 data channels, each with two dedicated preamplifiers: one, with selectable gain to cover the low and medium energy ranges of up to $20 \mathrm{MeV}$ and $1 \mathrm{GeV}$, respectively; the other, a low-gain amplifier covering the full dynamic range of $20 \mathrm{GeV}$. These allow implantation and decay signals to be processed differently, since passing implantation and decay signals through the same amplifier and the same gain would not provide useful data. A schematic showing the functionality of a single ASIC channel is 
shown in Fig. 1.

The energy deposited by the heavy ion implant can be as high as $20 \mathrm{GeV}$, whilst the subsequent decay products have energies in the range of tens of $\mathrm{keV}$ to $\mathrm{MeV}$. The ASICs must be capable of performing spectroscopy quality measurements over these energy ranges within $\mu$ s of the initial multi-GeV implant. From Fig. 1, it can be seen that the low/intermediate energy range amplifier is directly connected to the analogue input (the signal from a single strip of the DSSSD), whilst the high energy amplifier is connected via a diode link. This serves to separate the two energy ranges and allow for the automatic selection of the correct amplifier to suit the energy deposited in the silicon during either an implantation or decay event.

The high charge collected after an implantation event leads to the saturation of the low/intermediate energy amplifier and fluctuation in the input voltage. This forward biases the link diode to the high energy amplifier, with the connection maintained by the CMOS switch, until the remainder of the charge is integrated by the amplifier. The signal is shaped and stored by a peak-hold before being read out.

Separation of the high and low/intermediate energy channels helps achieve the fast overload recovery of the system required for decay spectroscopy measurements as the control logic can initiate the reset of the low/intermediate energy amplifier while the high-energy signal is still being processed. This gives sensitivity to decay products with a few microseconds of the initial heavy-ion implant.

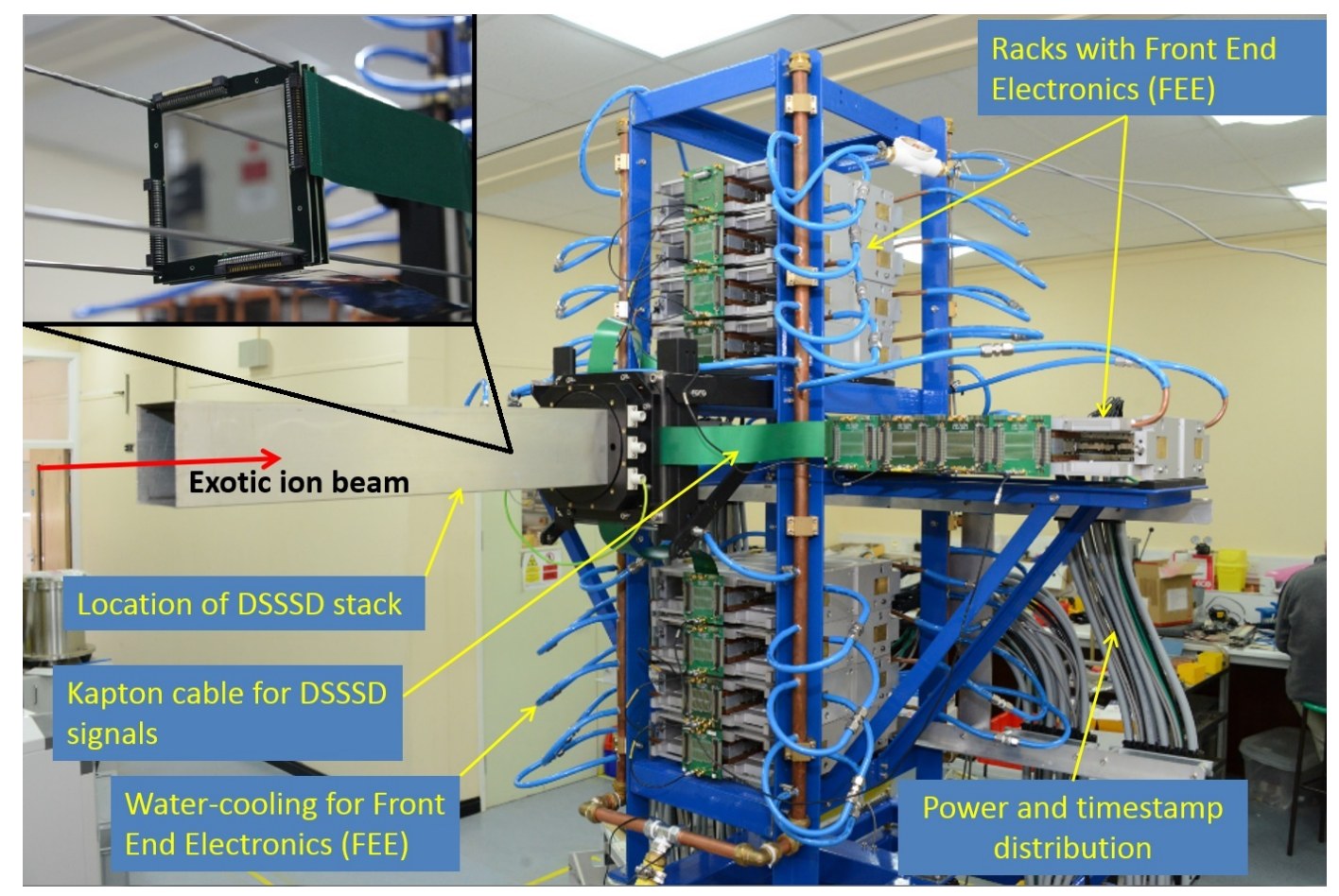

Figure 2: A photograph of the fully constructed AIDA assembly. Shown is the blue support assembly, which contains the aluminium cooling crates that house the FEE modules and provides support for the power and data cables. The long 'nose' containing the DSSSD stack can be seen at the front of the assembly. Inset: one of the MSL type BB18 DSSSDs in position on guide rails along the nose. 
Front end electronics (FEE) The front end electronics (FEE) cards are designed to support 64 channels of instrumentation. The FEE cards contain: multiple analogue-to-digital converters (ADCs) for use in signal processing; a field-programmable gate array (FPGA) for control, signal processing and event building; and supporting electronics and connections to enable stable operation and the output of data to a remote tape server. As each FEE card runs as a separate triggerless data acquisition system (DAQ), reading data from just 64 channels, dead-time is vastly reduced compared to current generation detectors dealing with high pixelation. This allows for much higher implantation rates and, combined with the high yields currently being reached at beam fragmentation facilities, enables AIDA to probe much more exotic regions of the chart of nuclides. Fig. 2 shows the full AIDA assembly, including the supporting framework and FEE modules housed in aluminium cooling racks.

Data output As each FEE card operates as an independent DAQ system, their individual data streams must be merged into one continuous time-ordered stream for analysis. Data are output from each FEE card via a gigabit Ethernet port, with each data item marked by a 48-bit timestamp. Using the timestamp information, data can be synchronised between the ASICs within each FEE card and between each FEE card within the wider system. This can then be extended to include data streams from other detector systems operated alongside AIDA. Finally, the individual data items are merged into one continuous time-ordered data stream.

\section{Current Progress and outlook}

System tests have been carried out using a variety of methods, including pulser tests of electronic components, radioactive source tests and in-beam tests at the Radioactive Ion Beam Factory (RIBF) at RIKEN, Tokyo. Fig. 3 shows data obtained through source tests and the implantation of stable heavy ions with production AIDA hardware. Frame a shows the detector response to conversion electrons emitted from a ${ }^{207} \mathrm{Bi}$ source, using the low energy $(20 \mathrm{MeV})$ preamplifier channel. The $22 \mathrm{keV}$ FWHM resolution observed is representative of what should be expected from AIDA in future experimental campaigns. Frame b shows a spectrum obtained through the implantation of heavy stable ions in the detector, demonstrating the large range of energies the AIDA electronics are capable of processing.

With promising progress being made on all fronts, AIDA is planned for use at RIBF[12, 13] throughout 2015-2016 with two main focuses: $\beta$-decay half-life and decay spectroscopy measurements with the EURICA $\gamma$-ray detector, and measurements of the $\beta$-delayed neutron emission probabilities as part of the BRIKEN ( $\beta$-delayed neutrons at RIKEN) collaboration.

Looking further ahead, AIDA is also planned for use at the FAIR Super-FRS upgrade of GSI as part of the DESPEC (Decay Spectroscopy) experiment which will probe nuclear structure and provide nuclear astrophysics data for unstable nuclei.

The compact front end and fast overload recovery capabilities make AIDA suitable for a wide range of uses including the decay spectroscopy of exotic proton and $\alpha$-particle emitters (with much shorter half-lives than exotic neutron-rich nuclei), as well as possible nuclear structure studies. However, the current focus of AIDA is that of $\beta$-decay spectroscopy. 

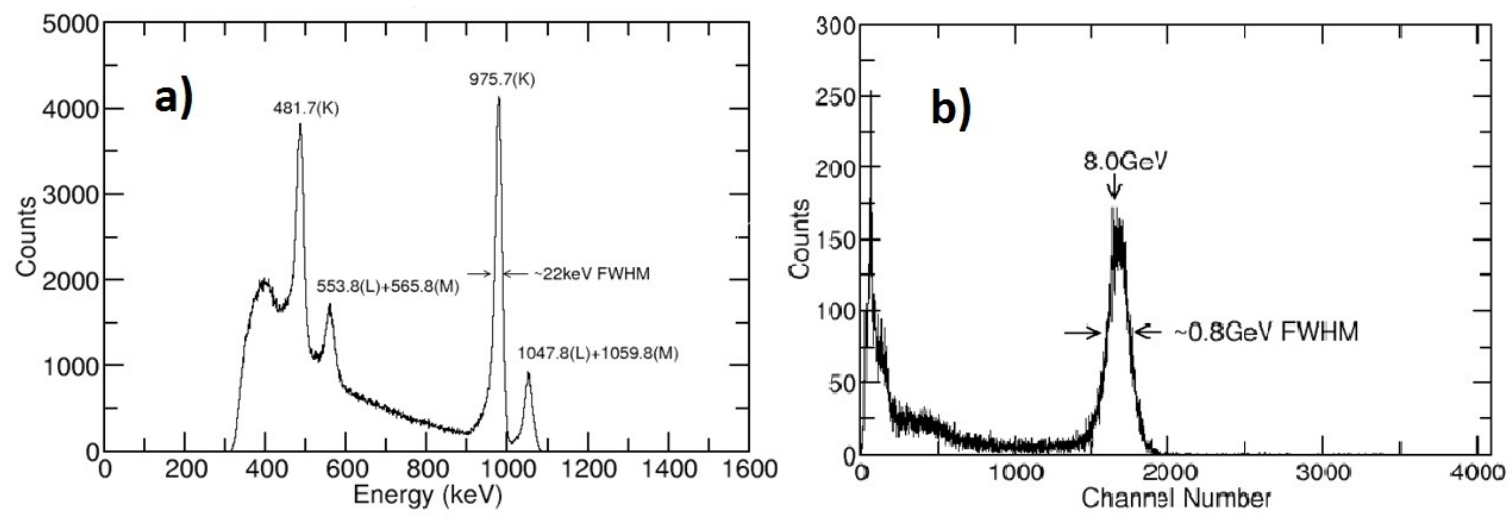

Figure 3: Plots showing the response of production AIDA hardware to source tests with ${ }^{207} \mathrm{Bi}$ (a) and heavy, stable ion implants (b). Plot a shows peaks from the conversion electrons of ${ }^{207} \mathrm{Bi}$, with the electron energies labelled, highlighting the $22 \mathrm{keV}$ FWHM resolution that should be expected of the low-energy amplifiers in future experimental campaigns.

\section{References}

[1] E. M. Burbidge, G. R. Burbidge, W. A. Fowler, and F. Hoyle Rev. Mod. Phys., vol. 29, pp. 547-650, 1957.

[2] A. G. W. Cameron Publ. Astron. Soc. Pac., pp. 201-222, 1957.

[3] G. Wallerstein, I. Iben, P. Parker, A. M. Boesgaard, G. M. Hale, A. E. Champagne, C. A. Barnes, F. Käppeler, V. V. Smith, R. D. Hoffman, et al. Rev. Mod. Phys., vol. 69, no. 4, p. 995, 1997.

[4] F. Käppeler Prog. Part. Nucl. Phys., vol. 43, pp. 419-483, 1999.

[5] M. Busso, R. Gallino, and G. Wasserburg Annu. Rev. Astron. Astro., vol. 37, no. 1, pp. 239-309, 1999.

[6] Y.-Z. Qian Prog. Part. Nucl. Phys., vol. 50, no. 1, pp. 153-199, 2003.

[7] M. Arnould, S. Goriely, and K. Takahashi Phys. Rep., vol. 450, no. 4, pp. 97-213, 2007.

[8] M. Mumpower, J. Cass, G. Passucci, R. Surman, and A. Aprahamian AIP Advances, vol. 4, no. 4, p. 1009, 2014.

[9] A. Arcones and G. Martínez-Pinedo Phys. Rev. C, vol. 83, no. 4, p. 045809, 2011.

[10] T. Davinson. http://www2.ph.ed.ac.uk/ td/AIDA/welcome.html, Aug 2014.

[11] D. Braga, P. J. Coleman-Smith, T. Davinson, I. H. Lazarus, R. Page, and S. Thomas in ANIMMA 2nd International Conference, pp. 1-5, IEEE, 2011.

[12] S. Nishimura, Z. Li, H. Watanabe, K. Yoshinaga, T. Sumikama, T. Tachibana, K. Yamaguchi, M. Kurata-Nishimura, G. Lorusso, Y. Miyashita, et al. Phys. Rev. Lett., vol. 106, no. 5, p. 052502, 2011.

[13] S. Nishimura Prog. Theor. Exp. Phys., vol. 2012, no. 1, p. 03C006, 2012. 